\title{
The World's first global telescope network at your fingertips
}

\author{
Edward L. Gomez ${ }^{1,2}$ and Haley L. Gomez ${ }^{2}$ \\ ${ }^{1}$ Las Cumbres Observatory Global Telescope, Inc., \\ 6740 Cortona Dr, Suite 102, Goleta, CA 93117, USA \\ email: egomez@lcogt.net \\ ${ }^{2}$ School of Physics and Astronomy, Cardiff University, \\ The Parade, Cardiff, UK, CF24 3AA \\ email: haley.gomez@astro.cf.ac.uk
}

\begin{abstract}
We discuss the creation of the World's largest homogeneous telescope network. We summarize both the scientific and education programmes, and outline why this organization provides unique opportunities for anyone interested in astronomy, whether they are professionals or amateurs, experienced or novice.
\end{abstract}

Keywords. telescopes:robotic, activities: education, outreach.

\section{Introduction}

Las Cumbres Observatory Global Telescope Network (LCOGT) is a privately operating foundation, building a global network of telescopes for scientific research and investigation-lead education. The global headquarters (California, USA) are home to the majority of the research astronomers, and the mechanical, electrical and software engineers responsible for building the network telescopes. In addition, there is a smaller centre in the UK whose primary task is telescope management and operations, but also has a team of software engineers.

There are two aspects to our mission:

(a) To establish and build a durable scientific institution dedicated to time-domain astrophysics.

(b) To use astronomical research to inspire critical thinking and technical understanding in young people and learners of all ages.

In this review we outline the plans for the telescope network, and we discuss some of the main science programmes and corresponding educational resources carried out by LCOGT astronomers and collaborators.

\section{Global Telescope Network}

LCOGT's network currently consists of two operating $2 \mathrm{~m}$ telescopes which are undergoing improvements and upgrades during 2008-2009 and will eventually total 27 telescopes at sites across the globe (Table 1, Fig. 1). At present the confirmed sites are Haleakala (Maui, USA), Siding Spring (New South Wales, Australia) and Cerro Tololo (Chile). There is still negotiations on site locations, and the exact sites may change as the network evolves. Each Telescope in the network will operate robotically, without any need for a human prescence at site during normal operations. Each site has weather and telemetry sensors which automatically inform the telescope and enclosure whether conditions are 
Table 1. General properties of the network classes.

\begin{tabular}{lccccc}
\hline $\begin{array}{l}\text { Size } \\
\text { primary }\end{array}$ & Quantity & Location & Clusters & $\begin{array}{c}\text { Field of View } \\
\text { arcmin }\end{array}$ & $\begin{array}{c}\text { Pixel Size } \\
\text { arcsecs }\end{array}$ \\
\hline $2 \mathrm{~m}$ & 2 & Haleakala, Sliding Spring & & 4.7 & 0.14 \\
$1 \mathrm{~m}$ & 12 & various & 2 or 4 & 26.6 & TBC \\
$0.4 \mathrm{~m}$ & $10-15$ & various & 2 or 3 & 29.4 & 0.58 \\
\hline
\end{tabular}

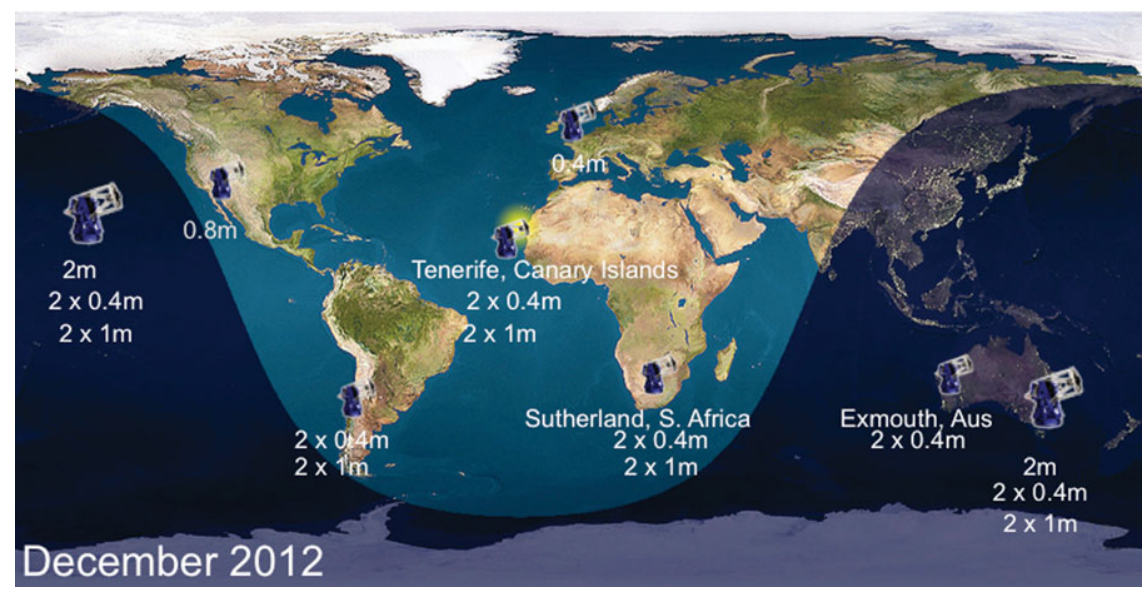

Figure 1. LCOGT aims to keep astonomers in the dark. This is the projected distribution of the network by the end of 2012 .

suitable for observing. At present, observations can be made either by remote control, where an observer directly controls the telescope, or by robotic scheduler, where observations are submitted to a queue. The following list details the requirements the network must fulfill in order to achieve the mission of the project:

- rapid response to targets of opportunities, e.g. microlensing events, supernovae and gamma-ray bursts

- scheduled event monitoring (a central scheduler)

- moving sources, e.g. near Earth objects (NEOs)

- periodic sampling of interesting objects, e.g. X-ray binaries

- central online archive of data

- automated calibration pipeline.

The network of telescopes will operate as a "single global telescope", with a standarized and homogeneous photometric system with identical filters, CCDs, calibration and reduction processes. To fullfill the science objectives, the global telescope network will include three classes of telescope: $0.4,1.0 \mathrm{~m}$ and $2.0 \mathrm{~m}$. The details of each class are listed below and in Table 1. There may also be occasional additions to the network which do not fit within these classes. One such addition is the Sedgwick Reserve Observatory (California, USA), which has $0.8 \mathrm{~m}$ aperture and is a custom design system. It has recently been installed and is now under going commissioning in the Santa Ynez Valley, California.

2m: There are two LCOGT telescopes in this class, Faulkes Telescope North and Faulkes Telescope South. They were manufactured by Telescope Technologies Ltd (which has now become the UK centre for telescope management for LCOGT). The Faulkes 

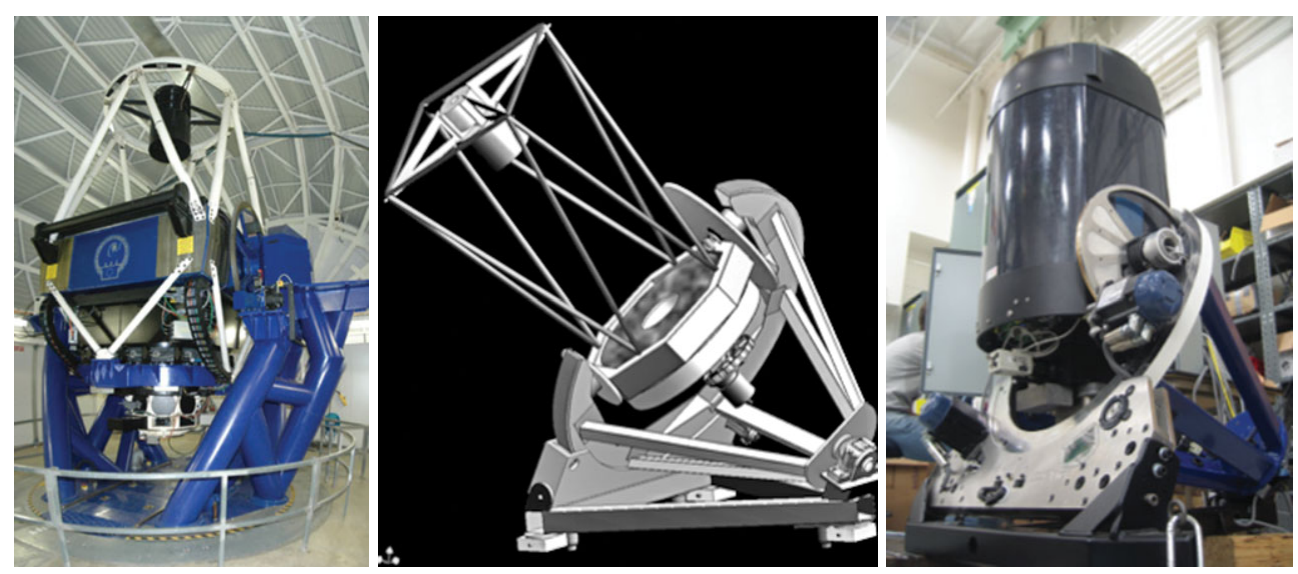

Figure 2. The $2 \mathrm{~m}$ telescope, Faulkes Telescope North (FTN) located in Hawaii. (b) Design for $1.0 \mathrm{~m}$ telescope. (c) $0.4 \mathrm{~m}$ telescope currently being tested in the workshop (Goleta, California).

Telescopes (FTN and FTS, Fig. 2a) are located in Hawaii and Australia respectively. They are a Ritchey-Chretien design, with an Alt/Az mount. The $2 \mathrm{~m}$ class is used regularly by LCOGT astronomers, and also by UK and Hawaii schools. Some of the specifics for this class are listed in Table 1 .

$\underline{1 m}$ : This class of telescope (Fig. $2 \mathrm{~b}$ ) is currently in design phase with prototype testing beginning in June 2009. Deployment to Cerro Tololo (Chile) is planned for late in 2009 or early 2010. The current optical tube design is reminiscent of a scaled down version of the $2 \mathrm{~m}$ class, being similar to a Ritchey-Chretien system. Unlike the $2 \mathrm{~m}$ class, each $1 \mathrm{~m}$ will be mounted on a C-ring, equatorial mount and not an Alt/Az mount (the latter system makes it very difficult to track objects that pass directly overhead). This increases the stability and reliability for tracking objects (particularly through the zenith) but also increases the telescope footprint.

$0.4 \mathrm{~m}$ : This class of telescopes is currently in production at LCOGT's Santa Barbara headquarters (Fig. 2c). The first prototype is working and image quality has been validated. These telescopes are based around a modified Meade OTA telescope, 40 of which have been acquired. These have all been tested and the 12 with the best quality optics will be installed on a custom C-ring, equatorial mount, with high specification CCD cameras. They will be installed in Aqawan enclosures (specially designed by LCOGT staff, but inspired by the MONET enclosure at McDonald Observatory) in pairs, except at Haleakala and Siding Spring, where they will be installed inside the clam-shell enclosures which house the $2 \mathrm{~m}$ telescopes. During 2009 there will be a total of six $0.4 \mathrm{~m}$ telescopes installed across four sites. Two will be installed in each of the Faulkes Telescope enclosures (Haleakala and Siding Spring), one will go to Liverpool (to be used as a sandbox for software testing and future modifications) and another will go to Cerro Tololo (Chile).

This class of telescope will be almost exclusively available to non-professional astronomers to use, whether they are school students, amateur astronomers, or members of the general public. They will be installed at so-called dark sites (along with the professional classes), there will be at least two per site and all will have a professional set of instruments. The $0.4 \mathrm{~m}$ class will therefore provide high quality data and high availability, making them perfect for education and outreach. 
The unique aspect of LCOGT is the scale of the telescope network. Many heterogeneous networks already exist (e.g. eMerlin, RoboNet), but the LCOGT network will be the largest which is operated by a single organization. Each LCOGT telescope or node will be designed specifically to fit into the 'single' network. This approach has the advantage that LCOGT can define the network standard for instrumentation, calibration, operations and maintanence. The driving philosophy is to have a network in which data from one site can be directly compared and used in conjunction with data from another site. This is a significant engineering challenge (both physically and in the software required), and a key element in its success will be the creation of a central observation sequencer, which will efficiently and reliably schedule observations.

\section{Science programme}

Having a largely custom-made, homogeneous network spread strategically across the globe in the northern and southern hemisphere means that LCOGT can respond rapidly to unexpected and exciting events in the night sky. The distribution of telescope sites (see Fig. 1) allows scientist to follow the time variations of single objects over days or weeks. The network will also ensure that whenever an short-lived event occurs, LCOGT will have at least one telescope in the dark and ready to detect it. This is reflected in the LCOGT science mission statement: "to establish and build a durable scientific institution dedicated to time-domain astrophysics."

LCOGT has an active research programme already underway with astronomers involved in a number of world-class research projects including the hunt for exoplanets and nearby supernovae. Some details on our science projects are listed below and in Figs. 3, 4 and 5 .

Extrasolar planets: The discovery of planets orbiting distant stars is one of the most exciting fields in astronomy today and interest in it is continuously growing. New research fields are fast emerging, such as the field of Astrobiology which focuses on the study of proto-stellar systems, planets and ultimately life itself. Over the past few years, systems with massive planets at very small orbital radii have proved to be quite common despite being generally unexpected. It was originally thought that large gas-giant planets cannot form close to their host stars since the stellar wind would have blown away most of the surrounding gas in the disc to a much farther distance, but the theoretical formation models have had to be revised in the light of the new discoveries.

The current number of confirmed exoplanets exceeds 270 (20 of which have been found by LCOGT astronomers using SuperWASP e.g. Pollacco et al. (2008), with the vast majority of these discovered by radial velocity surveys and transit events (e.g. Fig. 3a \& b). These surveys are biased towards the detection of systems with massive planets (several times the mass of Jupiter) in small orbits. A useful technique with the potential to detect Earth-mass planets from the ground, is so-called microlensing. Gravitational microlensing (Fig. 4a) relies on one-off chance meetings to discover exoplanets. If a star moves in front of a more distant star (relative to an observer on earth), the light from the distant star is bent by the gravitational pull of the closer star. As the closer star moves in front of the distant star the magnified image of the distant star gets brighter, reaches a peak and then dims in a smooth transition. If the closer star has one or more planets in orbit around it, the shape of the brightening and dimming magnified image is not smooth, but has an extra 'blip' (the exact shape of the 'blip' depends mostly on how near to eclipsing the more distant star the closer one comes). Fig. $4 \mathrm{~b}$ shows how the brightness of the magnified star changes with time; the blip on the otherwise smooth curve, is due to the 

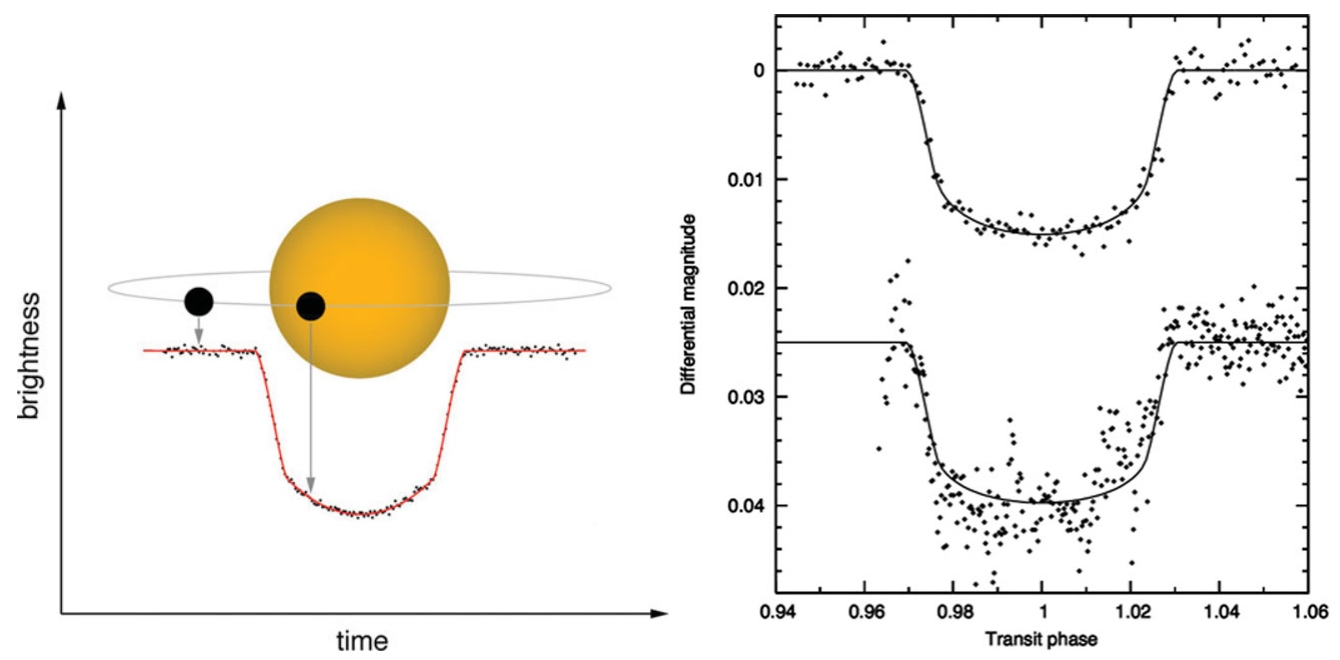

Figure 3. (a) A schematic of exoplanet WASP-10b crossing in front of its star. The brightness of the star decreases as the planet moves in front and behind, allowing astronomers to measure the size of the planet. (Credit: SuperWASP consortium.) (b) The light curve of WASP-5b obtained with the Euler telescope (top) and FTS (bottom). The solid line is the model fit to the data and implies that WASP-5b is a hot-Jupiter planet (Anderson et al. 2008).

presence of a planet with mass about 5 times the mass of the Earth, lying at an orbital radius which would fall between Mars and Jupiter in our own Solar System.

Extragalactic transients: Supernovae are exploding stars so bright they can be seen from distant galaxies, hundreds of millions to billions of light years away (Fig. 5a). Type Ia supernovae are used to map out the history of the expansion of the universe and have revealed that the universe is expanding at an ever-accelerating rate due to the presence of a mysterious source of energy (Bazin et al. 2009). The LCOGT network will study the lightcurves of nearby supernovae (e.g. Fig. 5b) in combination with more distant supernovae discovered by the Supernova Legacy Survey (SNLS, a recently-completed five year program involving the Canada-France-Hawaii Telescope, Keck, Gemini, and VLT) to determine the nature of dark energy. LCOGT scientists will also study other types of supernovae (e.g. Howell et al. 2009), which provide information on the explosive deaths of massive stars and the births of black holes and pulsars.

Clusters: Clusters are an ideal astrophysical site for testing theories on star formation and evolution. There are many science projects within this subset including monitoring the magnetic field cycles and angular momentum of pre-main sequence stars and understanding variable massive stars. One major study with LCOGT telescopes will look into the physics of massive-star formation, which remains an outstanding problem within astronomy. For stars with masses greater than 20 times that of the Sun, the brevity of the formation phase and the presence of gas and dust in their birth clouds, make observations of high mass protostars highly challenging. We can turn the problem on its head by using observed characteristics of massive stars to constrain the physics set at formation (e.g. whether high mass stars live in binary systems like low mass stars). 

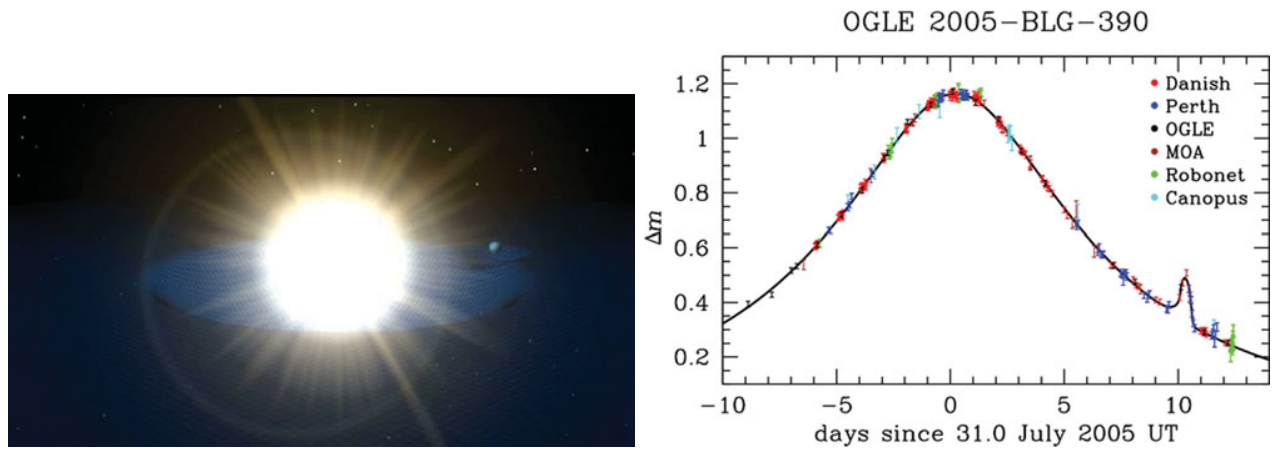

Figure 4. (a) Schematic of a microlensing event (LCOGT/J.Yardley). The combined gravitational field of the star and planet causes a blip in brightness as the system passes in front of a distant star (b) The microlensing event OGLE 2005-BLG-390 (Beaulieu et al. 2006).
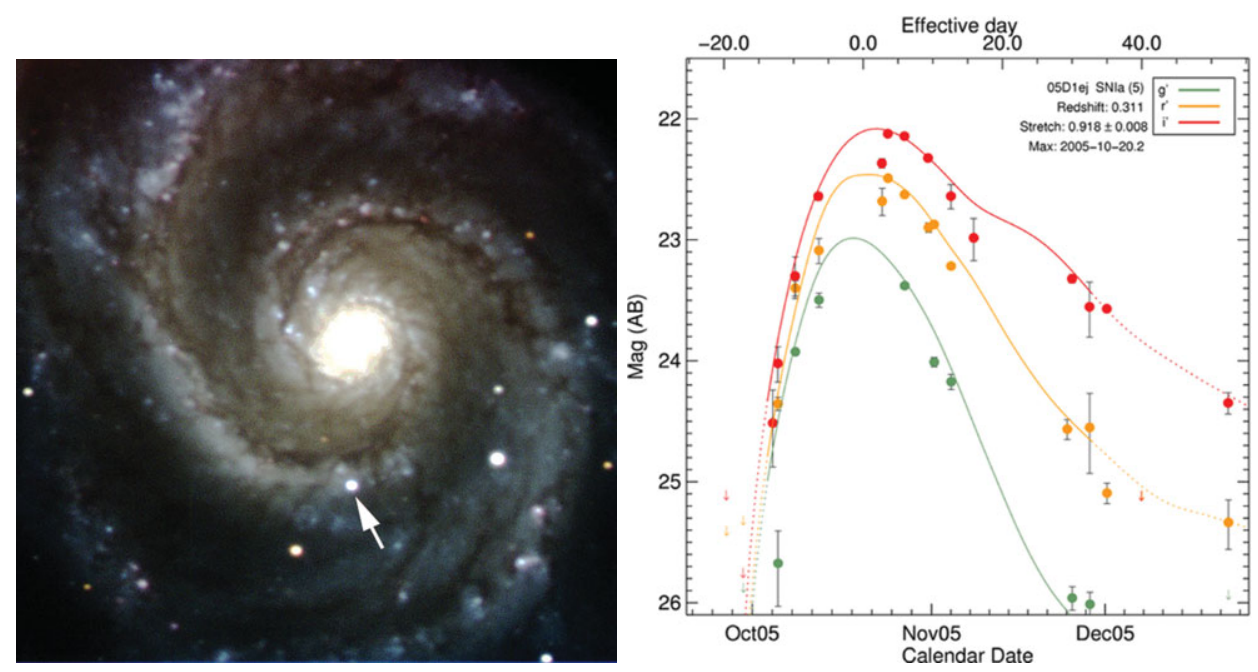

Figure 5. (a) FTN image of SN2007cs (located by arrow) in the galaxy M51. (b) Optical light curve of a Type Ia supernova in different filters (Sullivan et al. 2009, in preparation).

\section{Education Programme}

Telescope use at present, is only open to educational users (schools and astronomy clubs) in the UK and Hawaii. Our education programme aims to use the telescopes to excite, inspire and encourage learners of all ages. The global nature of LCOGT makes personal contact with the majority of our target audience impossible. For this reason, most of the education work will be carried out over the internet, via LCOGT's website: lcogt.net. Currently lcogt.net has a large number of online tools, including "how-to" articles and tutorials (about astronomy in general), and related activities which are all available at http://lcogt.net/education. The education website will continue to be developed during 2009-2010 to include more activities, ways to collaborate on projects (we have already introduced a novel 'groups' feature on the site), observation planning tools, interactive applications on astronomy, and astronomer interviews/vodcasts. Specifically, we aim to: "use astronomical research to inspire critical thinking and technical understanding in young people".

Through our expanding set of 'how-to' guides, people can learn more about general astronomy, and about using the telescope network to gain experience in planning an 

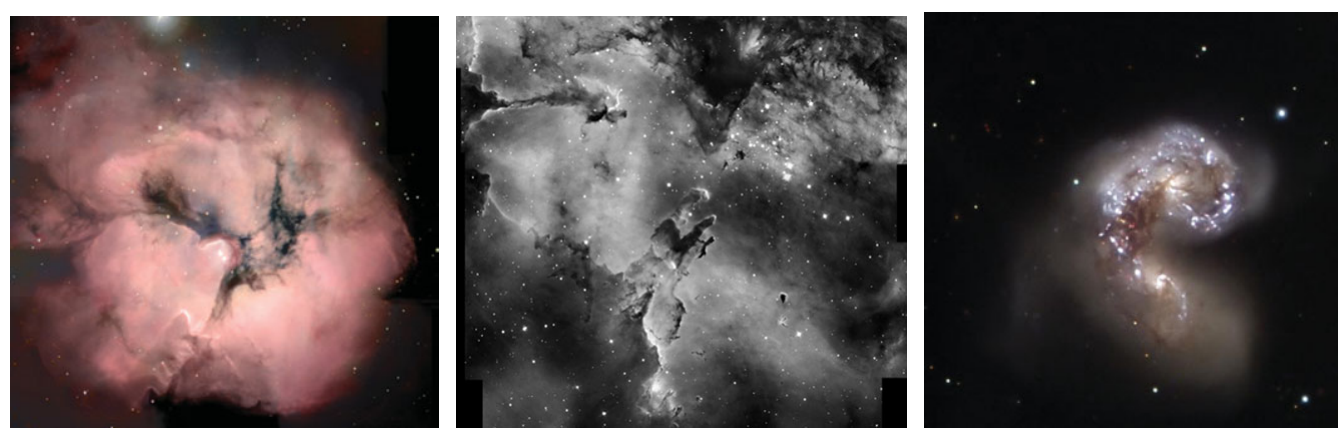

Figure 6. Striking images taken with FTN. M20 (Image processing: Nik Symanek). The Horsehead nebula observed by FTN using 27 individual images (each 60s in $\mathrm{H}_{\alpha}$ ) mosaiced together. 3-colour image (R, B, V filters) of the interacting galaxies NGC4038/9. (Image processing: D Duggan for LCOGT.)

observing session, image processing e.g. creating stunning colour images (Fig.6) using different filter sets or performing photometry and data analysis. Experienced users can be involved in more demanding education programmes, which are designed to have an advanced investigative nature. Most of our core education programmes are designed to reflect the topics in our science research programme.

Asteroids, Comets and Near-Earth Objects: In the UK, this programme is run in conjunction with the Faulkes Telescope Project (an official LCOGT education partner) and allows users to search for asteroids and NEOs. Since this programme began in 2004, schools have contributed observations whose results have been published in leading astronomical journals, including the recently discovered, fastest rotating asteroid (Fig. 7). $2008 \mathrm{HJ}$ is an object the size of a small tennis court but is rotating at an amazing 42.7 times a second. School students have also discovered and been involved in the discovery of 4 asteroids and renamed one of these, asteroid 2003 SM15 as Snowdonia. LCOGT is also a member of the Pan-STARRS collaboration whose major science goal is to track down NEOs.

Supernovae: There are two components to this educational programme. The first involves observing historical supernovae in the Milky Way, searching for the remnants left behind after the original explosion. Users can compare images taken over a period of several years to determine the age of the remnant and the expansion rate of the gases ejected in the supernova event. Users can also compare the remnant in three different 'colours' i.e. wavelengths including X-ray and radio data. This project is suitable for people to undertake without needing to control the telescope as the LCOGT public archive can be used and sample data is also provided. The second part of this programme is run in collaboration with European Hands on Universe (Eu-HOU), and involves discovering and making follow-up observations of recent nearby supernovae to help characterize the nature of the explosion. This part requires telescope access and use of the supernova portal available at eu-hou.net.

Exoplanets: This is the newest programme to be offered. The LCOGT astronomers select potential exoplanet candidates and put them into a urgent target list (available and updated daily on lcogt.net). Once the system has been observed, an automated data pipeline checks the observations and analyses them for the presence of an exoplanet: education users can now be involved in the discovery of new planets! 


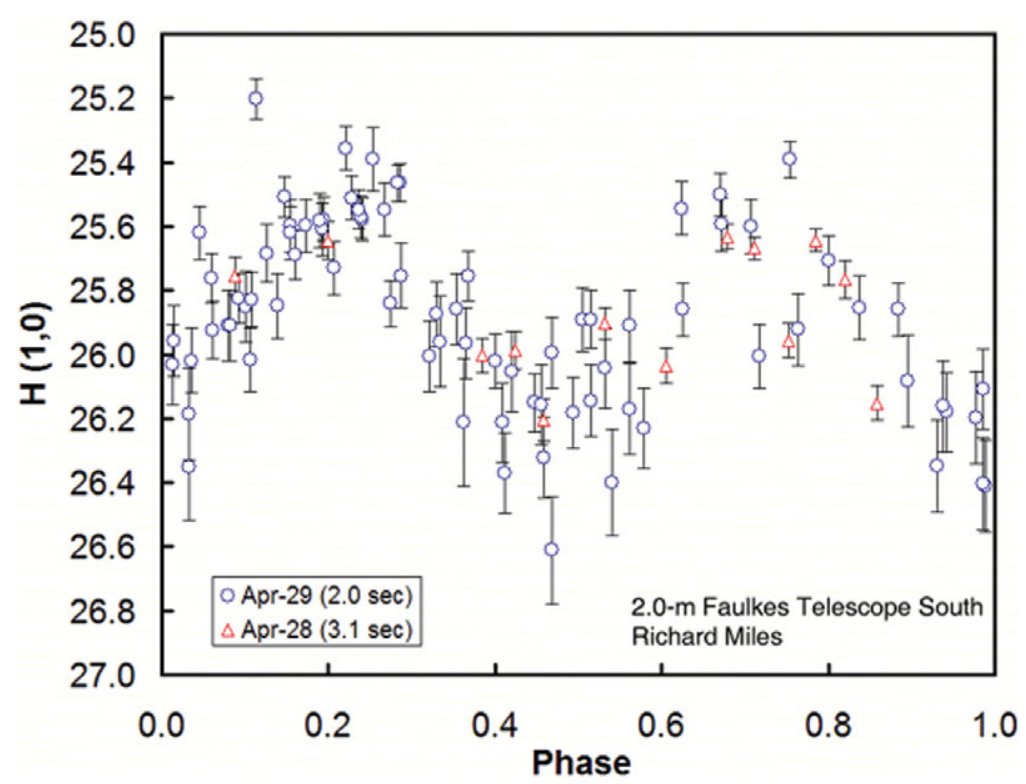

Figure 7. Light curve of the fastest rotating asteroid discovered with FTS observations analysed by Richard Miles, BAA (including observations taken by school children).

We hope to be able to start offering access to users from the USA mainland from early 2010, with access to anyone in the world once the network fully complete.. We have started pilot programmes with groups in Poland and Russia, where professional astronomers and amateurs volunteered to support local astronomy projects. We aim to make access to the network completely free of charge. For more details about our education programmes see lcogt.net/education.

\section{Summary}

LCOGT is the World's largest global telescope network with a homogeneous network of 27 telescopes due to be in place by 2013. These telescopes will provide unprecedented research opportunities for astronomers but will also provide an unique experience to anyone with an interest in astronomy. Both science and education programmes will have large amounts of telescope time available to them.

For the latest news or to get involved in our science and education programmes, subscribe to our email newsletter or RSS feed at http://lcogt.net.

\section{Acknowledgements}

Thanks to the LCOGT astronomers. We acknowledge the support of our education partners, particularly the Faulkes Telescope Project (faulkes-telescope.com), University of Hawaii, European Hands-on-Universe, Astronomical Society of the Pacific, Astronomia.pl, and the British Council. 


\section{References}

Anderson, D. R., et al. 2008, MNRAS, 387, L4

Bazin, G., et al. 2009, A\& $A, 499,653$

Beaulieu, J.-P., et al. 2006, Nature, 439, 437

Howell, D. A., et al. 2009, ApJ, 691, 661

Pollacco, D., et al. 2008, MNRAS, 385, 1576 